\title{
INVARIANT SUBSPACES WITH ZERO DENSITY SPECTRUM
}

\section{O.A. KRIVOSHEEVA}

\begin{abstract}
In the paper we show that each analytic solution of a homogeneous convolution equation with the characteristic function of minimal exponential type is represented by a series of exponential polynomials in its domain. This series converges absolutely and uniformly on compact subsets in this domain. It is known that if the characteristic function is of minimal exponential type, the density of its zero set is equal to zero. This is why in the work we consider the sequences of exponents having zero density. We provide a simple description of the space of the coefficients for the aforementioned series. Moreover, we provide a complete description of all possible system of functions constructed by rather small groups, for which the representation by the series of exponential polynomials holds.
\end{abstract}

Keywords: A series of exponential monomials, relatively small clusters, basis, convex domain.

Mathematics Subject Classification: 30D10

Let $\Lambda=\left\{\lambda_{k}, n_{k}\right\}_{k=1}^{\infty}$ be a multiple sequence of complex numbers with non-decreasing absolute values, that is, $\lambda_{k} \in \mathbb{C}, n_{k}$ is a natural number called the multiplicity of the point $\lambda_{k},\left|\lambda_{k+1}\right| \geqslant$ $\left|\lambda_{k}\right|, k=1,2, \ldots$, and $\left|\lambda_{k}\right| \rightarrow \infty$ as $k \rightarrow \infty$. Let $B(z, r)$ be the ball of radius $r$ centered at a point $z$. We denote by $n(r, \Lambda)$ the number of the points $\lambda_{k}$ counting the multiplicities located in the ball $B(0, r), r>0$. The upper density of the sequence $\Lambda$ is

$$
\bar{n}(\Lambda)=\varlimsup_{r \rightarrow \infty} \frac{n(r, \Lambda)}{r} .
$$

We consider the sequence of simple points $\Lambda=\left\{\lambda_{k}, 1\right\}_{k=1}^{\infty}=\left\{\lambda_{k}\right\}_{k=1}^{\infty}$ and the series

$$
\sum_{k=1}^{\infty} d_{k} e^{\lambda_{k} z} .
$$

G. Pólya [1] proved that for the zero density $\bar{n}(\Lambda)=0$ each sum of the series has a convex domain; this function is analytic. In work [2] G. Pólya generalized this result. Consider the homogeneous convolution equation

$$
M_{f}(g)=\int_{\mathbb{C}} g(z+w) d \mu(z) \equiv 0,
$$

where $\mu$ is a complex-valued compactly supported measure and

$$
f(\lambda)=\int_{\mathbb{C}} e^{\lambda z} d \mu(z)
$$

is the Laplace transform of the functional generating the convolution operator called the characteristic function of the operator $M_{f}$. Assume that $f(\lambda)$ is a function of the minimal exponential

O.A. Krivosheeva, Invariant subspaces with zero density SPECTRum.

(c) Krivosheeva O.A. 2017.

Submitted October 31, 2016. 
type, that is, for each $\varepsilon>0$ there exists a constant $C(\varepsilon)>0$ such that $\ln |f(\lambda)| \leqslant C(\varepsilon)+\varepsilon|\lambda|$, $\lambda \in \mathbb{C}$. Then the operator $M_{f}$ is defined in the space of functions $g$ analytic in the vicinity of the origin; each $g$ is analytic in its neighbourhood. It was shown in work [2] that under the mentioned condition, each analytic solution to equation (2) has a convex domain.

Let $\Lambda=\left\{\lambda_{k}, n_{k}\right\}_{k=1}^{\infty}$ be a sequence of zeroes and their multiplicities for the function $f$. We note that if $f(\lambda)$ is a function of minimal exponential type, the density $\bar{n}(\Lambda)$ of its zero set is zero, see [3, Ch. I, Sect. 11, Lindelöf theorem].

In work [4] Valiron showed that each analytic solution of equation (2) is represented as

$$
g(z)=\lim _{m \rightarrow \infty} \sum_{\left|\lambda_{k}\right|<r_{m}} \sum_{n=0}^{n_{k}-1} d_{k, n} z^{n} e^{\lambda_{k} z}
$$

in its domain, where $\left\{r_{m}\right\}$ is an unboundedly increasing sequence of positive numbers such that the absolute value of the function $f$ has appropriate lower bounds on the circumferences $|\lambda|=r_{m}, m=1,2, \ldots$ The system of the functions $\mathcal{E}(\Lambda)=\left\{z^{n} \exp \left(\lambda_{k} z\right)\right\}, k \geqslant 1, n=\overline{0, n_{k}-1}$, is called the set of elementary solutions to equation (2).

In the present work, we strengthen this result by Valiron. We show that each analytic solution to equation (2) with the characteristic function $f(\lambda)$ of minimal exponential type is represented by the series

$$
g(z)=\sum_{m=1, j=1}^{\infty, N_{m}} d_{m, j} e_{m, j}(z)
$$

in its domain, where $\left\{e_{m, j}(z)\right\}_{m=1, j=1}^{\infty, N_{m}}$ is a fixed system of the functions, each being a finite linear combination of the functions in the system $\mathcal{E}(\Lambda)$. At that, series (4) converges absolutely and uniformly on compact sets. We provide a simple description of the space of the coefficients $d=\left\{d_{m, j}\right\}_{m=1, j=1}^{\infty, N_{m}}$. Moreover, we provide a complete description of all possible systems $\left\{e_{m, j}(z)\right\}$ constructed by relatively small groups $U_{m}$ of the exponents $\lambda_{k}$ of the functions in $\mathcal{E}(\Lambda)$, for which representation (4) holds.

Let $D$ be a convex domain in $\mathbb{C}$ and $H(D)$ stand for the space of analytic in $D$ functions with the topology of uniform convergence on compact subsets $D$. By $H^{*}(D)$ we denote the strongly dual space for $H(D)$ called the space of analytic functionals. Given a sequence $\Lambda=\left\{\lambda_{k}, n_{k}\right\}_{k=1}^{\infty}$, by $W(\Lambda, D)$ we denote the closure of the linear span of the system $\mathcal{E}(\Lambda)$ in the space $H(D)$. The subspace $W(\Lambda, D)$ is closed and invariant w.r.t. the differentiation operator. If $a \in \mathbb{C}$, we let

$$
W(\Lambda, a)=\bigcup_{D \ni a} W(\Lambda, D)
$$

where $D$ ranges in the set of all convex neighbourhoods of the point $a$. We equip the space $W(\Lambda, a)$ with the topology of the inductive limit.

We note that according to the above mentioned result by Valiron, the space of analytic solution to equation (2) with the characteristic function $f(\lambda)$ of minimal exponential type is embedded into the space $W(\Lambda, 0)$.

Lemma 1. Let $\Lambda=\left\{\lambda_{k}, n_{k}\right\}_{k=1}^{\infty}$ and $a \in \mathbb{C}$. Assume that each function in $W(\Lambda, a)$ has a convex domain. Then $\bar{n}(\Lambda)=0$

Proof. Let $\bar{n}(\Lambda)>0$. We consider first the case of simple $\lambda_{k}$, that is, $\Lambda=\left\{\lambda_{k}, 1\right\}_{k=1}^{\infty}$. It follows from [3, Ch. IV, Sect. 7, Thm. 18] that in this case the system $\mathcal{E}(\Lambda)$ is complete in the space of the functions analytic in the circle of radius $\bar{n}(\Lambda) / e$ centered at $a$. Therefore, the embedding $H(B(a, \bar{n}(\Lambda) / e)) \subset W(\Lambda, a)$ holds. Since not all functions in $H(B(a, \bar{n}(\Lambda)) / e)$ has a convex domain, this contradicts the assumption of the lemma. 
Let us show that the case of multiple $\lambda_{k}$ is reduced to the above studied case. In order to do this, we introduce an auxiliary sequence $\tilde{\Lambda}=\left\{\lambda_{k, j}, 1\right\}_{k=1, j=1}^{\infty, n_{k}}$. We arbitrarily choose the points $\lambda_{k, j}$ by the conditions: 1) all $\lambda_{k, j}$ are different; 2) $\left|\lambda_{k, j}-\lambda_{k}\right|<1, j=\overline{1, n_{k}}, k \geqslant 1$. Then the systems $\mathcal{E}(\Lambda)$ and $\mathcal{E}(\tilde{\Lambda})$ are complete or incomplete simultaneously in each convex domain $D$.

Indeed, let $D$ be a convex domain. We recall that the system $\mathcal{E}(\Lambda)(\mathcal{E}(\tilde{\Lambda}))$ is incomplete in $D$ if and only if there exists an entire function $f(\tilde{f})$ of exponential type satisfying two conditions: 1) the conjugate diagram $f(\tilde{f})$ lies in $D ; 2)$ the function $f(\tilde{f})$ vanishes at the points $\lambda_{k}\left(\lambda_{k, j}\right)$ withe the multiplicities at least $n_{k},\left(n_{k}=1\right)$. Thus, let $\mathcal{E}(\Lambda)$ be incomplete in $H(D)$. Then there exists a function $f$ possessing properties 1), 2). It is represented as $f=h g$, where $h$ and $g$ are entire functions of first order, not necessarily of finite, that is, of exponential type. At that, the function $h$ vanishes only at the points $\lambda_{k}$ with the multiplicities $n_{k}$, and the functions $g$ does at other zeroes of the function $f$ with the needed multiplicity. Let $\tilde{h}$ be an entire function of first order vanishing only at the points $\lambda_{k, j}$ with the multiplicity one. We consider the function $\tilde{f}=\tilde{h} g$. By the choice of the function $\lambda_{k, j}$, Theorem in work [5] implies that $\tilde{f}$ is an entire function of exponential type, whose conjugate diagram coincides with the conjugate diagram of the function $f$, that is, it is located in $D$. By construction, the function $\tilde{f}$ vanishes at the points $\lambda_{k, j}$ with the multiplicity at least one. Thus, $\tilde{f}$ satisfies Conditions 1$), 2$ ), that is, $\mathcal{E}(\Lambda)$ is incomplete in $H(D)$. The opposite statement can be proved in the same way. The proof is complete.

Hereafter we assume that the sequence $\Lambda=\left\{\lambda_{k}, n_{k}\right\}_{k=1}^{\infty}$ has the zero density $\bar{n}(\Lambda)=0$. Let us construct the aforementioned system of functions $\left\{e_{m, j}(z)\right\}$.

Let $U=\left\{U_{m}\right\}_{m=1}^{\infty}$ be a partition of $\Lambda$ into finite groups $U_{m}$. We renumber the terms of $\Lambda$. The points $\lambda_{k}$ in the group $U_{m}$ are denoted by $\lambda_{m, l}$, and their multiplicities are denoted by $n_{m, l}$. The first index coincides with the index of the group, while the other ranges from 1 to $M_{m}$, where $M_{m}$ the number of the points $\lambda_{k}$ in the group $U_{m}$. We let $N_{m}=\sum_{l=1}^{M_{m}} n_{m, l}$. We shall say that groups $U_{m}=\left\{\lambda_{m, l}\right\}_{l=1}^{M_{m}}$ are relatively small if

$$
\lim _{m \rightarrow \infty} \max _{1 \leqslant j, l \leqslant M_{m}} \frac{\left|\lambda_{m, j}-\lambda_{m, l}\right|}{\left|\lambda_{m, 1}\right|}=0 .
$$

This implies easily that

$$
\lim _{m \rightarrow \infty} \max _{1 \leqslant j, l \leqslant M_{m}} \frac{\left|\lambda_{m, j}\right|}{\left|\lambda_{m, 1}\right|}=1
$$

By the latter identity and the condition $\bar{n}(\Lambda)=0$ we obtain

$$
\lim _{m \rightarrow \infty} \frac{N_{m}}{\left|\lambda_{m, 1}\right|}=0
$$

Following work [6], by the system $\mathcal{E}(\Lambda)=\left\{z^{n} \exp \left(\lambda_{k} z\right)\right\}$ we construct a new system of functions $\mathcal{E}(\Lambda, U)=\left\{e_{m, j}(z)\right\}_{m=1, j=1}^{\infty, N_{m}}$. Let $\Gamma_{m}$ be a contour (simple closed continuous rectifiable curve) enveloping the points of the group $U_{m}$ and

$$
\omega_{m}(\lambda)=\prod_{l=1}^{M_{m}}\left(\lambda-\lambda_{m, l}\right)^{n_{m, l}}, \quad m=1,2, \ldots
$$

We let

$$
P_{m}(\lambda, z)=\frac{1}{2 \pi i} \int_{\Gamma_{m}} \frac{\exp (z \zeta)\left(\omega_{m}(\zeta)-\omega_{m}(\lambda)\right)}{(\zeta-\lambda) \omega_{m}(\zeta)} d \zeta, \quad m=1,2, \ldots
$$


This formula defines the known interpolation polynomial of degree at most $N_{m}-1$, whose values and the derivatives up to the order $n_{m, l}-1$ at the points $\lambda_{m, l}$ coincide with the corresponding values of the function $\exp (z \zeta)$ and of its derivatives, that is,

$$
P_{m}^{(n)}\left(\lambda_{m, l}, z\right)=z^{n} \exp \left(\lambda_{m, l} z\right), \quad l=1,2, \ldots, M_{m}, \quad n=0,1, \ldots, n_{m, l}-1 .
$$

We let

$$
e_{m, j}(z)=P_{m}^{(j-1)}\left(\lambda_{m, 1}, z\right), \quad j=1, \ldots, N_{m}, \quad m=1,2, \ldots
$$

Let $U$ be the trivial partition of the sequence $\Lambda$, that is, each group $U_{m}$ consists of one point $\left.\lambda_{m, 1}=\lambda_{m}\right)$. It is easy to see that the system $\mathcal{E}(\Lambda, U)$ coincides with $\mathcal{E}(\Lambda)$.

We consider also the system of the functions

$$
e_{m, j}^{\prime}(z)=\sum_{k=1}^{N_{m}} a_{m, j, k} e_{m, k}(z), \quad m=1,2, \ldots, \quad j=1, \ldots, N_{m} .
$$

We denote the set $\left\{e_{m, j}^{\prime}\right\}_{m=1, j=1}^{\infty, N_{m}}$ by $\mathcal{E}(\Lambda, U, \mathbb{A})$, where $\mathbb{A}=\left\{\mathcal{A}_{m}\right\}_{m=1}^{\infty}$ and $\mathcal{A}_{m}=\left(a_{m, j, k}\right)$ is the matrix of transition from $\left\{e_{m, k}\right\}_{k=1}^{N_{m}}$ to $\left\{e_{m, j}^{\prime}\right\}_{j=1}^{N_{m}}$. We shall say that the system $\mathcal{E}(\Lambda, U, \mathbb{A})$ is normalized if $\max _{1 \leqslant k \leqslant N_{m}}\left|a_{m, j, k}\right|=1, j=1, \ldots, N_{m}, m=1,2, \ldots$

For each convex domain $D \subset \mathbb{C}$ we fix a sequence of convex compact sets $\mathcal{K}(D)=\left\{K_{p}\right\}_{p=1}^{\infty}$, which strictly exhausts it, that is, $K_{p} \subset$ int $K_{p+1}, p \geqslant 1$, int denotes the interior of a set and $D=\cup_{p=1}^{\infty} K_{p}$.

Let $\Lambda=\left\{\xi_{m}\right\}_{m=1}^{\infty}$ be a sequence of complex numbers such that $\left|\xi_{m}\right| \rightarrow \infty$ as $k \rightarrow \infty$ and $e_{m}$ are entire functions $m \geqslant 1$. We shall say [7] that $\left\{e_{m}\right\}_{m=1}^{\infty}$ is an almost exponential sequence with the exponents $\left\{\xi_{m}\right\}$ if for each convex domain $D \subset \mathbb{C}$ two conditions hold:

1) for each $p \geqslant 1$ there exist a number $a>0$ and an index $s$ such that

$$
\sup _{w \in K_{p}}\left|e_{m}(w)\right| \leqslant a \exp \left(H_{K_{s}}\left(\xi_{m}\right)\right), \quad m \geqslant 1 ;
$$

2) for each $p \geqslant 1$ there exist a number $a>0$ and an index $s$ such that

$$
b \exp \left(H_{K_{p}}\left(\xi_{m}\right)\right) \leqslant \sup _{w \in K_{s}}\left|e_{m}(w)\right|, \quad m \geqslant 1 .
$$

Here $H_{M}(\lambda)$ stands for the support function of the set $M$, more precisely, for the complex conjugation of the set $M$ :

$$
H_{M}(\lambda)=\sup _{w \in M} \operatorname{Re}(\lambda w), \quad \lambda \in \mathbb{C} .
$$

Conditions 1) and 2) mean that the sequence $\left\{e_{m}\right\}_{m=1}^{\infty}$ is some sense similar to the sequence of the exponentials $\left\{\exp \left(\xi_{m} z\right)\right\}_{m=1}^{\infty}$.

In view of identity (5), by Theorem 6 in work [8] we have the following statement.

Lemma 2. Let $\Lambda=\left\{\lambda_{k}, n_{k}\right\}_{k=1}^{\infty}$ be such that $\bar{n}(\Lambda)=0$ and $U=\left\{U_{m}\right\}_{m=1}^{\infty}$ be a partition of $\Lambda$ into relatively small groups. Then each normalized system $\mathcal{E}(\Lambda, U, \mathbb{A})$ defined by the formulae (6), (7) is an almost exponential sequence with the exponents $\left\{\lambda_{m, 1}\right\}$ (more precisely, with the exponents $\lambda_{m, j}^{\prime}$, where $\lambda_{m, j}^{\prime}=\lambda_{m, 1}, j=\overline{1, N_{m}}$ ).

Let $\mathcal{E}(\Lambda, U, \mathbb{A})=\left\{e_{m, j}^{\prime}\right\}_{m=1, j=1}^{\infty, N_{m}}$. We consider the series

$$
\sum_{m=1, j=1}^{\infty, N_{m}} d_{m, j} e_{m, j}^{\prime}(z)
$$

Lemma 2 implies immediately that for the above series, Theorem 3.1 from work [9] is true; the Abel theorem for the series of exponential polynomials. In order to formulate, we shall need some notations. 
Let $E$ be a set in $\mathbb{C}, \Theta$ be a closed subset of the unit circumference $\mathbb{S}$ centered at zero. The convex hull $\Theta$ of $E$ is the set

$$
E(\Theta)=\left\{z \in \mathbb{C}: \operatorname{Re}(z \xi)<H_{E}(\xi), \xi \in \Theta\right\} .
$$

The convex hull $\Theta$ of a set coincides with its usual convex hull, more precisely, with the interior of this convex hull if $\Theta=\mathbb{S}$. We also observe that the interior $E$ is located in $E(\Theta)$. According Lemma 2.1 in work [9], the set $E(\Theta)$ is a convex domain.

Let $\Lambda=\left\{\lambda_{k}, n_{k}\right\}_{k=1}^{\infty}$. By the symbol $\Theta(\Lambda)$ we denote the set of all partial limits of the sequence $\left\{\lambda_{k} /\left|\lambda_{k}\right|\right\}_{k=1}^{\infty}$ except for the point $\lambda_{k}=0$ if it is present. It is obvious that $\Theta(\Lambda)$ is a closed subset of the circumference $\mathbb{S}$.

Theorem 1. [9] Let $\Lambda=\left\{\lambda_{k}, n_{k}\right\}_{k=1}^{\infty}$ be such that $\bar{n}(\Lambda)=0, U=\left\{U_{m}\right\}_{m=1}^{\infty}$ be the partition of $\Lambda$ into relatively small groups and $\mathcal{E}(\Lambda, U, \mathbb{A})=\left\{e_{m, j}^{\prime}\right\}_{m=1, j=1}^{\infty, N_{m}}$ be the normalized system. Assume that the terms of series (8) are bounded on each compact set of a bounded set $E \subset \mathbb{C}$. Then series (8) converges absolutely and uniformly in each compact set in the convex domain $D=E(\Theta(\Lambda))$.

Since $\Lambda=\left\{\lambda_{k}, n_{k}\right\}_{k=1}^{\infty}$ has the zero density, the system $\mathcal{E}(\Lambda)$ is incomplete in the space $H(D)$ for each convex domain $D \subset \mathbb{C}$. Indeed, the functional $\mu \in H^{*}(D)$, whose Laplace transform is equal to

$$
f(z)=\exp (a z) \prod_{k=1}^{\infty}\left(1-\left(\frac{z}{\lambda_{k}}\right)^{2}\right)^{n_{k}}
$$

vanishes at all functions of the system $\mathcal{E}(\Lambda)$ and $\mu \neq 0, a$ is some point in the domain $D$. By the incompleteness of $\mathcal{E}(\Lambda)$, the system $\mathcal{E}(\Lambda, U)=\left\{e_{m, j}(z)\right\}_{m=1, j=1}^{\infty, N_{m}}$ possesses a biorthogonal sequence of the functionals $\left\{\mu_{m, j}\right\}_{m=1, j=1}^{\infty, N_{m}} \subset H^{*}(D)$ for each convex domain $D$, that is, $\mu_{m, j}\left(e_{l, p}\right)=1$ if $m=l, j=p$ and $\mu_{m, j}\left(e_{l, p}\right)=0$ otherwise, see [10, Sect. 2].

Let $a \in \mathbb{C}$ and a function $g \in W(\Lambda, a)$ is expanded into a series converging uniformly on each compact set in the open set $E \subset \mathbb{C}$ :

$$
g(z)=\sum_{m=1, j=1}^{\infty, N_{m}} d_{m, j} e_{m, j}(z) .
$$

By Theorem 1, this series converges uniformly on each compact set in the convex domain $D=E(\Theta(\Lambda))$. Hence, the coefficients are uniquely determined by the formulae $d_{m, j}=\mu_{m, j}(g)$, $m \geqslant 1, j=\overline{1, N_{m}}$, where $\left\{\mu_{m, j}\right\} \subset H^{*}(D)$ is the system of functionals biorthogonal to $\mathcal{E}(\Lambda, U)$.

Let $a \in \mathbb{C}$. We shall say that the system $\mathcal{E}(\Lambda, U, \mathbb{A})$ is a basis in the subspace $W(\Lambda, a)$ if each its element $g$ is uniquely expanded into series (8), which converges uniformly on the compact sets in the domain of the function $g$.

By Theorem 1, the convergence domain of series (8) is convex. This is in view of (5), by Theorem 2.6 in work [10] we obtain the following result.

Theorem 2. Let $\Lambda=\left\{\lambda_{k}, n_{k}\right\}_{k=1}^{\infty}$ be such that $\bar{n}(\Lambda)=0$ and $U=\left\{U_{m}\right\}_{m=1}^{\infty}$ be the partition of $\Lambda$ into relatively small groups. If for some set of the matrices $\mathbb{A}=\left\{\mathcal{A}_{m}\right\}_{m=1}^{\infty}$ the system $\mathcal{E}(\Lambda, U, \mathbb{A})$ is a basis in the subspace $W(\Lambda, a)$, then $\mathcal{E}(\Lambda, U)$ is also a basis in $W(\Lambda, a)$.

Thus, by means of Theorem 2, the problem on existence of a basis in the subspace $W(\Lambda, a)$ of form $\mathcal{E}(\Lambda, U, \mathbb{A})$ constructed by relatively small groups is related to the basicity of a particular system of functions $\mathcal{E}(\Lambda, U)$.

Let us find out the conditions under which the system $\mathcal{E}(\Lambda, U)$ is a basis in the subspace $W(\Lambda, a)$. In order to do this, we shall need a scalar characteristics of the sequence $\Lambda$ called the condensation index. 
Assume that the sequence $\Lambda=\left\{\lambda_{k}, n_{k}\right\}_{k=1}^{\infty}$ is partitioned into the groups $U=\left\{U_{m}\right\}_{m=1}^{\infty}$, where $U_{m}=\left\{\lambda_{m, v}\right\}_{v=1}^{M_{m}}$. We let

$$
q_{\Lambda, U}^{m}(z, \delta)=\prod_{\lambda_{k, v} \in B\left(\lambda_{m, 1}, \delta\left|\lambda_{m, 1}\right|\right), k \neq m}\left(\frac{z-\lambda_{k, v}}{3 \delta\left|\lambda_{k, v}\right|}\right)^{n_{k, v}}, \quad m \geqslant 1 .
$$

The absolute value of the function $q_{\Lambda, U}^{m}(z, \delta)$ can be interpreted as a measure of accumulation of the points $\lambda_{k, v} \in B\left(\lambda_{m, 1}, \delta\left|\lambda_{m, 1}\right|\right), k \neq m$, near $z$. If they are absent, we let $q_{\Lambda, U}^{m}(z, \delta) \equiv 1$. Following [11] and [10], we introduce the condensation index of the groups $U_{m}$ of the sequence $\Lambda$ :

$$
S_{\Lambda}(U)=\lim _{\delta \rightarrow 0} \lim _{m \rightarrow \infty} \min _{\lambda_{m, v} \in B\left(\lambda_{m, 1}, \delta\left|\lambda_{m, 1}\right|\right)} \frac{\ln \left|q_{\Lambda, U}^{m}\left(\lambda_{m, v}, \delta\right)\right|}{\left|\lambda_{m, v}\right|} .
$$

We note that the coefficient 3 in the definition of $q_{\Lambda, U}^{m}$ is chosen just for convenience, see [11, Rem. 1 to Thm. 5.1]. This ensures the inequality $S_{\Lambda}(U) \leqslant 0$. If $U$ is the trivial partition, that is, each group $U_{m}$ consists of one point, then $S_{\Lambda}(U)$ coincides with the quantity $S_{\Lambda}$ introduced in work [11]. The identity $S_{\Lambda}(U)=0$ means that the groups $U_{m}$ are in some sense separated.

Let $\Lambda=\left\{\lambda_{k}, n_{k}\right\}_{k=1}^{\infty}$ have the zero density. Then the sequence $\left\{\lambda_{k}, n_{k}\right\}_{k=1}^{\infty} \cup\left\{-\lambda_{k}, n_{k}\right\}_{k=1}^{\infty}$ is a properly distributed set [3, Ch. II, Sect. 1]. Then the sequence is proper in the sense of Definition 3.1 in work [10]. Then according Lemma 3.5 in work [10], we have the following statement.

Lemma 3. Let $\Lambda=\left\{\lambda_{k}, n_{k}\right\}_{k=1}^{\infty}$ be such that $\bar{n}(\Lambda)=0$. Then there exists a partition $U$ of sequence $\Lambda$ into relatively small groups such that $S_{\Lambda}(U)=0$.

Now we are in position to formulate and prove a result solving the issue on the basis in the subspace $W(\Lambda, a)$.

Theorem 3. Let $a \in \mathbb{C}, \Lambda=\left\{\lambda_{k}, n_{k}\right\}_{k=1}^{\infty}$ be such that $\bar{n}(\Lambda)=0$ and $U=\left\{U_{m}\right\}_{m=1}^{\infty}$ be the partition of $\Lambda$ into relatively small groups. The following statements are equivalent:

1) the system $\mathcal{E}(\Lambda, U)$ is a basis in the subspace $W(\Lambda, a)$;

2) $S_{\Lambda}(U)=0$.

Proof. Assume that Statement 1) is true. Let $D$ be a bounded convex domain containing the point $a$. Then in view of 1$)$, by the definition of the subspace $W(\Lambda, a)$ we find that each function $g \in W(\Lambda, D)$ is uniquely expanded into series (10), which converges uniformly on compact sets in the domain of the function $g$. In particular, it converges uniformly on each compact set in $D$.

Thus, Statement 2) in Theorem 3.1 in work [12] is true. According this theorem, the identity $S_{\Lambda}(U)=0$ holds true.

Assume now that Statement 2) is true and $g$ is an arbitrary element in the subspace $W(\Lambda, a)$. According the definition of $W(\Lambda, a)$, there exists a bounded convex domain $D$ such that $g \in$ $W(\Lambda, D)$. Since $\bar{n}(\Lambda)=0$ and $S_{\Lambda}(U)=0$, Statement 5) in Theorem 3.1 in work [12] holds true. According this theorem, the function $g$ is expanded into series (10), which converges uniformly on compact sets in the domain $D$.

Let $G$ be an open set consisting of all points in the plane such that series (10) converges uniformly in some neighbourhood of such points. Theorem 1 implies that $G$ is a convex domain. The inclusion $D \subset G$ holds. This is why the function $g$ is analytically extended into the domain $G$ and is represented by series (10) converging uniformly on compact sets in $G$. In particular, $g \in W(\Lambda, G)$.

Let us show that $G$ is the domain of the function $g$. Assume the opposite, that is, there exists a boundary point $z_{0}$ of the domain $G$ and a number $r>0$ such that $g$ is analytically 
continued into the ball $B\left(z_{0}, r\right)$. We fix an arbitrary point $b$ in the intersection of the domain $G$ and the ball $B\left(z_{0}, r / 2\right)$. Then the ball $B(b, r / 2)$ contains a neighbourhood of the point $z_{0}$ and lies inside the ball $B\left(z_{0}, r\right)$.

Thus, the function $g$ is analytic in the ball $B(b, r / 2)$. Since $b \in G$, there exists $r^{\prime} \in(0, r / 2)$ such that the ball $B\left(b, r^{\prime}\right)$ lies in the domain $G$. By the belonging $g \in W(\Lambda, G)$ this yields: $g \in W\left(\Lambda, B\left(b, r^{\prime}\right)\right)$. The ball $B(b, r / 2)$ is the sum of two balls $B\left(b, r^{\prime}\right)$ and $B\left(0, r^{\prime \prime}\right)$, where $r^{\prime}+r^{\prime \prime}=r / 2$. Therefore, by Theorem 12.1 in work [13], the function $g$ is approximated by linear combinations of the system $\mathcal{E}(\Lambda)$ in the ball $B(b, r / 2)$, that is, $g \in W(\Lambda, B(b, r / 2))$.

As above, for the convex domain $\tilde{D}=B(b, r / 2)$, Statement 5$)$ of Theorem 3.1 in work [12] holds. According this theorem, the function $g$ is expanded into the series

$$
g(z)=\sum_{m=1, j=1}^{\infty, N_{m}} \tilde{d}_{m, j} e_{m, j}(z),
$$

which converges uniformly on compact sets in the domain $\tilde{D}$. Therefore, in the intersection $G \cap \tilde{D}$ we have both representations (10) and (11). It has been mentioned above that the system $\mathcal{E}(\Lambda, U)=\left\{e_{m, j}(z)\right\}_{m=1, j=1}^{\infty, N_{m}}$ possesses a biorthogonal sequence of functionals in each convex domain and, in particular, in $G \cap \tilde{D}$. This is why the representation by series (10) is unique, that is, the identities $d_{m, j}=\tilde{d}_{m, j}, m \geqslant 1, j=\overline{1, N_{m}}$, hold. This means that the function $g$ is expanded into series (10) in the union of the domains $G$ and $\tilde{D}$, which converges uniformly on compact sets in $G \cup \tilde{D}$. Since $G$ is a proper subset of the domain $G \cup \tilde{D}$, this contradicts the definition of the domain $G$.

Thus, our assumption is wrong, that is, $G$ is the domain of the function $g$. The proof is complete.

Under assumption that $\Lambda$ is partitioned into relatively small groups, Theorem 3 resolves the problem on the existence of a basis in the subspace $W(\Lambda, a)$. Moreover, it describes all appropriate $\left(S_{\Lambda}(U)=0\right)$ partitions of $\Lambda$ into relatively small groups.

Lemma 3 and Theorem 3 imply immediately the following statement.

Theorem 4. Let $a \in \mathbb{C}$ and $\Lambda=\left\{\lambda_{k}, n_{k}\right\}_{k=1}^{\infty}$ be such that $\bar{n}(\Lambda)=0$. Then there exists a partition $U$ of the sequence $\Lambda$ into relatively small groups such that the system $\mathcal{E}(\Lambda, U)$ is a basis $W(\Lambda, a)$.

Remark. We have mentioned above that the space of analytic solutions to equation (2) with the characteristic function $f(\lambda)$ of minimal exponential type is embedded into the space $W(\Lambda, 0)$, where $\Lambda$ is the multiple zero set of $f(\lambda)$; this is implied also by Theorem 6.1 in work [14], where the problem on spectral synthesis was solved for the spaces of solutions to homogeneous convolution equations. Therefore, Theorem 4 strengthens the aforementioned result by Valiron in work [4].

Lemma 1 and Theorems 1 and 4 yield the following result.

Theorem 5. Let $a \in \mathbb{C}$ and $\Lambda=\left\{\lambda_{k}, n_{k}\right\}_{k=1}^{\infty}$. The following statements are equivalent:

1) each function in $W(\Lambda, a)$ has a convex domain;

2) $\bar{n}(\Lambda)=0$.

Remark. Theorem 5 covers the aforementioned results by G. Pólya in works [1] and [2].

Let $U$ be the trivial partition of the sequence $\Lambda$. Then $\mathcal{E}(\Lambda, U)=\mathcal{E}(\Lambda), S_{\Lambda}(U)=S_{\Lambda}$; the quantity $S_{\Lambda}$ was introduced in work [11]. Therefore, Theorem 3 covers as a particular case the following solution of the problem on the fundamental principle for the subspace $W(\Lambda, a)$. 
Theorem 6. Let $a \in \mathbb{C}$ and $\Lambda=\left\{\lambda_{k}, n_{k}\right\}_{k=1}^{\infty}$ be such that $\bar{n}(\Lambda)=0$. The following statements are equivalent:

1) the system $\mathcal{E}(\Lambda)$ is a basis in the subspace $W(\Lambda, a)$;

2) $S_{\Lambda}=0$.

Remark. Theorem 6 is implied by Theorem 5.1 in work [15].

Now we are going to describe all possible bases in the subspace $W(\Lambda, a)$ for a fixed partition $U$ of the sequence $\Lambda$ into relatively small groups.

Let the system $\mathcal{E}\left(\Lambda, U, \mathbb{A}\right.$ ) (where $\mathbb{A}=\left\{\mathcal{A}_{m}\right\}_{m=1}^{\infty}$ ) be such that for each $m \geqslant 1$ the matrix $\mathcal{A}_{m}$ of transition from $\left\{e_{m, k}\right\}_{k=1}^{N_{m}}$ to $\left\{e_{m, j}^{\prime}\right\}_{j=1}^{N_{m}}$ is non-degenerate. By the symbol $\mathcal{B}_{m}=\left(b_{m, j, k}\right)$ we denote the matrix inverse for $\mathcal{A}_{m}$. We let

$$
\mathfrak{a}(\mathbb{A})=\varlimsup_{m \rightarrow \infty} \max _{1 \leqslant j, k \leqslant N_{m}} \frac{\ln \left|b_{m, j, k}\right|}{\left|\lambda_{m, 1}\right|} .
$$

By Theorem 2.7 in work [10] we obtain the following result.

Theorem 7. Let $a \in \mathbb{C}, \Lambda=\left\{\lambda_{k}, n_{k}\right\}_{k=1}^{\infty}$ be such that $\bar{n}(\Lambda)=0$ and $U=\left\{U_{m}\right\}_{m=1}^{\infty}$ be a partition of $\Lambda$ into relatively small groups. Assume that the system $\mathcal{E}(\Lambda, U)$ is a basis in the subspace $W(\Lambda, a)$, and the system $E(\Lambda, U, \mathbb{A})$ is normalized. Then the following statements are equivalent:

1) the system $\mathcal{E}(\Lambda, U, \mathbb{A})$ is a basis in $W(\Lambda, a)$;

2) $\mathfrak{a}(\mathbb{A})=0$.

In conclusion we describe the space of the coefficients of series (10), or equivalently, of series (8), representing the functions in the subspace $W(\Lambda, a)$.

Let $D$ be a convex domain in $\mathbb{C}, \mathcal{K}(D)=\left\{K_{p}\right\}_{p=1}^{\infty}, a \in \mathbb{C}$, let $\Lambda=\left\{\lambda_{k}, n_{k}\right\}_{k=1}^{\infty}$ be such that $\bar{n}(\Lambda)=0$ and $U=\left\{U_{m}\right\}_{m=1}^{\infty}$ be a partition of $\Lambda$ into relatively small groups. We introduce the Banach spaces of complex sequences:

$$
Q_{p}(\Lambda, U)=\left\{d=\left\{d_{m, j}\right\}_{m=1, j=1}^{\infty, N_{m}}:\|d\|_{p}=\sup _{m, j}\left|d_{m, j}\right| \exp H_{K_{p}}\left(\lambda_{k}\right)<\infty\right\}, \quad p \geqslant 1 .
$$

By the symbol $Q(\Lambda, U, D)$ we denote the projective limit of the spaces $Q_{p}, p \geqslant 1$, and the symbol $Q(\Lambda, U, a)$ stands for the inductive limit of the spaces $Q(\Lambda, U, D)$ over all convex domains $D$ containing the point $a$.

We define the operator $\mathfrak{B}$ acting on the space $Q(\Lambda, U, a)$ with values $W(\Lambda, a)$ by the rule: a sequence $d=\left\{d_{m, j}\right\} \in Q(\Lambda, U, a)$ is mapped into the sum $g(z)$ of series (10) if it converges in the topology of some space $H(D)$.

Theorem 2.2 in work [10] implies the following result.

Theorem 8. Let $a \in \mathbb{C}, \Lambda=\left\{\lambda_{k}, n_{k}\right\}_{k=1}^{\infty}$ be such that $\bar{n}(\Lambda)=0$ and $U=\left\{U_{m}\right\}_{m=1}^{\infty}$ be a partition of $\Lambda$ into relatively small groups. Then the following statements are equivalent:

1) the system $\mathcal{E}(\Lambda, U)$ is a basis in the subspace $W(\Lambda, a)$;

2) the operator $\mathfrak{B}$ is an isomorphism of linear topological spaces $Q(\Lambda, U, a)$ and $W(\Lambda, a)$.

\section{BIBLIOGRAPHY}

1. G. Pólya. Eine Verallgemeinerung des Fabryschen Lückensatzes // Nachr. Geselesch Wissensch. Gottingen. Math. Phys. Klas. 187-195 (1927).

2. G. Pólya. Untersuchungen über Lücken und Singularitäten von Potenzreihen // Math. Zeit. 29, 549-560 (1929).

3. B.Ya. Levin. Distribution of zeros of entire functions. Gostekhizdat, Moscow (1956). [Amer. Math. Soc., Providence, RI (1980).] 
4. G. Valiron. Sur les solutions des équations différentielles linéares d'ordre infinit et à coefficients constants // Ann. Sci. École Norm. Super. Sér. 3. 46, 25-53 (1929).

5. I.F. Krasichkov-Ternovskii. Comparison of entire functions of integral order by the distribution of their roots // Matem. Sborn. 70(112):2, 198-230 (1966). (in Russian).

6. A.S. Krivosheyev. Bases "in relatively small groups" // Ufimskij Matem. Zhurn. 2:2, 67-89 (2010). (in Russian).

7. A.S. Krivosheyev. An almost exponential basis // Ufimskij Matem. Zhurn. 2:1, 87-96 (2010). (in Russian).

8. A.S. Krivosheyev. An almost exponential sequence of exponential polynomials // Ufimskij Matem. Zhurn. 4:1, 88-106 (2012). [Ufa Math. J. 4:1, 82-100 (2012).]

9. O.A. Krivosheyeva. Convergence domain for series of exponential polynomials // Ufimskij Matem. Zhurn. 5:4, 84-90 (2013). [Ufa Math. J. 5:4, 82-87 (2013).]

10. A.S. Krivosheev, O.A. Krivosheeva. A basis in an invariant subspace of analytic functions // Matem. Sborn. 204:12, 49-104 (2013). [Sb. Math. 204:12, 1745-1796 (2013).]

11. A.S. Krivosheev. A fundamental principle for invariant subspaces in convex domains // Izv. RAN. Ser. Matem. 68:2, 71-136 (2004). [Izv. Math. 68:2, 291-353 (2004).] .

12. A.S. Krivosheev, O.A. Krivosheeva. Fundamental principle and a basis in invariant subspaces // Matem. Zamet. 99:5, 684-697 (2016). [Math. Notes. 99:5, 685-696 (2016).]

13. I.F. Krasichkov-Ternovskii. Invariant subspaces of analytic functions. III. On the extension of spectral synthesis // Matem. Sborn. 88(130):3, 331-352 (1972). [Math. USSR-Sb. 17:3, 327-348 (1972).]

14. I.F. Krasichkov-Ternovskii. Invariant subspaces of analytic functions. I. Spectral analysis on convex regions // Matem. Sborn. 88(130):1, 3-30 (1972). [Math. USSR-Sb. 16:4, 471-500 (1972).]

15. O.A. Krivosheyeva. Singular points of the sum of a series of exponential monomials on the boundary of the convergence domain // Alg. Anal. 23:2, 162-205 (2011). [St.-Petersburg Math. J. 23:2, 321-350 (2012).]

Olesya Alexandrovna Krivosheeva,

Bashkir State University,

Zaki Validi str. 32,

450076, Ufa, Russia

E-mail: kriolesya2006@yandex.ru 